URL: https://ami.uni-eszterhazy.hu

\title{
A survey on the global optimization problem using Kruskal-Wallis test
}

\author{
Viliam Ďuriš, Anna Tirpáková
}

\author{
Department of Mathematics \\ Constantine The Philosopher University in Nitra \\ Tr. A. Hlinku 1, 94974 Nitra, Slovakia \\ vduris@ukf.sk \\ atirpakova@ukf.sk \\ Submitted: April 1, 2020 \\ Accepted: May 26, 2020 \\ Published online: June 3, 2020
}

\begin{abstract}
The article deals with experimental comparison and verification of stochastic algorithms for global optimization while searching the global optimum in dimensions 3 and 4 of selected testing functions in Matlab computing environment. To draw a comparison, we took the algorithms Controlled Random Search, Differential Evolution that we created for this test and implemented in Matlab, and fminsearch function which is directly built in Matlab. The basic quantities to compare algorithms were time complexity while searching the considered area and reliability of finding the global optimum of the 1st De Jong function, Rosenbrock's saddle, Ackley's function and Griewangk's function. The time complexity of the algorithms was determined by the number of test function evaluations during the global optimum search and we analysed the results of the experiment using the "Kruskal-Wallis test" non-parametric method.

Keywords: global optimization; test functions; simplex; population; Controlled Random Search; Differential Evolution; fminsearch; Matlab, KruskalWallis test
\end{abstract}

MSC: 90C26, 62G09 


\section{Introduction}

In mathematics, we often solve a problem that we characterize as finding a minimum value of an examined function (so-called global optimization) $f: D \rightarrow R$ on a specific set $D \subseteq R^{d}, d \in N$. This minimum value (global minimum or global optimum) is one or more points from the smallest functional value set $D$, that is a set $\left\{x^{\prime} \in D: f\left(x^{\prime}\right) \leq f(x) \forall x \in D\right\}$ [8]. From mathematical analysis, we know the procedure for finding the extreme of a function when $d=2$ and there are the first and second function derivatives. However, finding a general solution to the problem formulated this way is very difficult (or even impossible) for any $d$ or if the function considered is multimodal or not differentiable [3]. Any deterministic algorithm addressing the generally formulated problem of global optimization is exponentially complex [2]. That is why we use the so-called randomly working (stochastic) algorithms to find a solution to this task, which, although not capable of finding a solution, are capable of finding a satisfactory solution to the problem within a reasonable time. Thus, for the same input problem, such an algorithm performs several different calculations and we aim to create conditions for the algorithm so that we reduce the probability of incorrect calculation as much as possible. Today, the use of stochastic algorithms, especially of the evolutionary type, is very successful in seeking global optimization functions [4]. Those are simple models of Darwin's evolutionary theory of populations development using selection (the strongest individuals are more likely to survive), crossing (from two or more individuals new individuals with combined parental properties will emerge) and mutation (accidental modification of information that an individual bears); to create a population with better properties. For some classes of evolution algorithms, the truly best "individuals" of the population are approaching the global optimum.

Experimental verification and comparison of algorithms on test functions offer us an insight into the performance and behavior of the used global optimization algorithms. Based on this, we can then decide which algorithm is most efficient and usable under the given conditions when solving practical tasks. The most basic test functions include the 1st De Jong function, Rosenbrock's saddle (2nd De Jong function), Ackley's function and Griewangk's function. Matlab source code of these functions can be found in [12].

In the Matlab environment, there are several implemented optimization functions, of which the fminsearch function [6] is very important. The fminsearch function serves for finding a global minimum of the function of multiple variables. The variables of the function, the global minimum of which we are looking for, are entered into the vector and, also, we specify the so-called start vector $x_{0}$, from which the search for the minimum will start. The start vector $x_{0}$ must be sufficiently close to the global minimum (not necessarily in unimodal functions only), because different estimates of the start vector may result in different local minima being found instead of the global one. Since the algorithm fminsearch is based on the so-called simplex method [9], it may happen that the solution will not be found at all. Otherwise, the fminsearch function returns the vector $x$, that is the point 
at which the global minimum of the given function is located.

Some optimization parameters for global minimum search can be specified in options structure using the function optimset [7]. Then the generic call command of the fminsearch function has the form

$$
[\mathrm{x}, \text { fval, exitflag, output }]=\text { fminsearch(fun, x0, options), }
$$

where fun is a string that records a given mathematical function, $x 0$ start vector, search setup options, $\mathrm{x}$ is the resulting vector of the global minimum, fval functional value at $x$, exitflag is a value, which specifies the type of search termination and output is a structure that contains the necessary optimization information (algorithm used, number of function evaluations, number of iterations).

For each algorithm, we need to distinguish four types of search termination. Type 1 is the correct completion of the algorithm when it is found (sufficiently close) to the global minimum, type 2 means that the algorithm is completed by reaching the maximum allowed number of iterations (although it converts to a global minimum), type 3 is an early convergence (the algorithm has completed searches in the local minimum) and type 4 means that browsing is completed by reaching the maximum allowed number of iterations, but no close point to the minimum has been found.

In order to determine the type of algorithm termination for the fminsearch function, it is possible to use the nested funcCount element of the output structure (which gives the number of function evaluations). You can also find out how to complete by using the exitflag element.

The Controlled Random Search (CRS) algorithm [11] works with a population of $N$ points in space $D$, from which a new point $y$ is generated with the so-called simplex reflex. Simplex $S=\left\{x_{1}, x_{2}, \ldots, x_{d+1}\right\}$ is a set of randomly selected $d+1$ space $D$ points. In simplex, we find the point $x_{h}=\max _{x \in S} f(x)$ with the highest functional value and, as the worst of simplex, we remove it. To the remaining $d$ points, we find their center of gravity

$$
g=\frac{1}{2} \sum_{x \in S}\left(x-x_{h}\right) .
$$

Reflexion means a point overturning $x_{h}$ around the center of gravity $g$ to obtain a point

$$
y=g+\left(g-x_{h}\right)=2 g-x_{h} .
$$

The simplest variant of the reflection algorithm can then be entered as a function in Matlab as follows: [12]

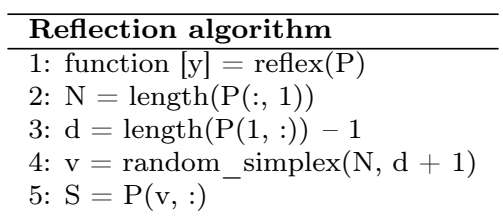




$$
\begin{aligned}
& \text { 6: }[\mathrm{x}, \mathrm{id}]=\max (\mathrm{S}(:, \mathrm{d}+1)) \\
& \text { 7: } \mathrm{x}=\mathrm{S}(\mathrm{id}, 1: \mathrm{d}) \\
& \text { 8: } \mathrm{S}(\mathrm{id},:)=[] \\
& \text { 9: } \mathrm{S}(:, \mathrm{d}+1)=[] \\
& \text { 10: } \mathrm{g}=\operatorname{mean}(\mathrm{S}) \\
& \text { 11: } \mathrm{y}=2^{*} \mathrm{~g}-\mathrm{x}
\end{aligned}
$$

where a random selection of a set $S$ is provided by the function

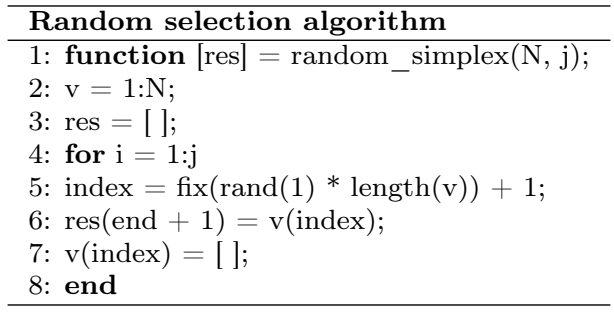

If the $f(y)<f\left(x_{h}\right)$, the point $y$ of the population replaces the point $x_{h}$ and we continue to do so. In case that $f(y) \geq f\left(x_{h}\right)$, simplex is reduced. By replacing the worst points of the population, this is concentrated around the lowest functional point being sought. However, the reflection does not guarantee that the newly generated point $y$ will be in the searched area $D$. Then, we flip all coordinates $y_{i} \notin\left\langle a_{i}, b_{i}\right\rangle, i=1, \ldots, d$, and inside of the searched area $D$ around the relevant side of the $d$-dimensional rectangular parallelepiped $D$. The algorithm of the so-called mirroring can be entered as a function in Matlab:

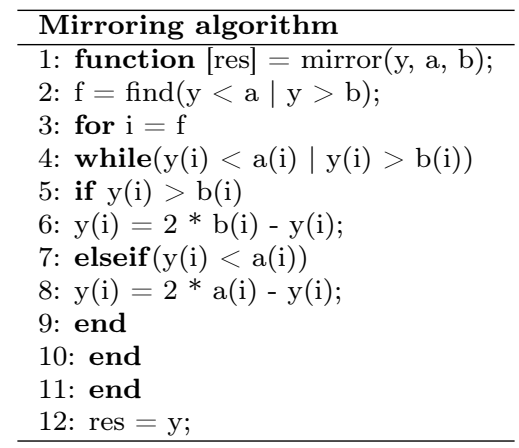

The algorithm's source text itself, Controlled Random Search, can be then written as an m-file crs.m in Matlab.

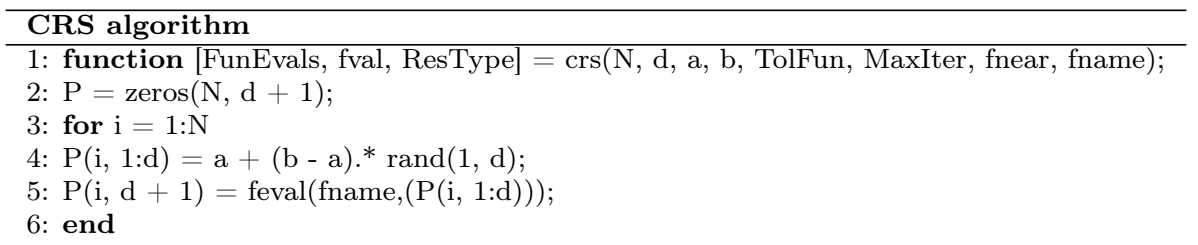


7: [fmax, indmax $]=\max (\mathrm{P}(:, \mathrm{d}+1))$;

8: [fval, indmin] $=\min (\mathrm{P}(:, \mathrm{d}+1))$;

9: FunEvals = N;

10: while (fmax - fval $>$ TolFun) \& (FunEvals $<\mathrm{d} *$ MaxIter)

11: $\mathrm{y}=\operatorname{reflex}(\mathrm{P})$;

12: $\mathrm{y}=\operatorname{mirror}(\mathrm{y}, \mathrm{a}, \mathrm{b})$;

13: fy $=$ feval(fname, y);

14: FunEvals $=$ FunEvals +1 ;

15: if $($ fy $<$ fmax $)$

16: $\mathrm{P}($ indmax,$:)=[\mathrm{y}$ fy $]$;

17: [fmax, indmax] $=\max (\mathrm{P}(:, \mathrm{d}+1))$;

18: [fval, indmin] $=\min (\mathrm{P}(:, \mathrm{d}+1))$;

19: end

20: end

21: if fval $<=$ fnear

22: if (fmax - fval) $<=$ TolFun

23: ResType $=1$;

24: else

25: ResType $=2$;

26: end

27: elseif (fmax - fval) $<=$ TolFun

28: ResType $=3$;

29: else

30: ResType $=4$;

31: end

The FunEvals variable is the counter of the number of algorithms' function evaluations. As the previous selection $N$ of population points results in $N$ function evaluation, it must be preset to the value $N$. Line 10 represents a search termination condition $\left(f_{\max }-f_{\min }<\epsilon\right) \vee($ FunEvals $>$ MaxIter $* d)$ where $d$ is the dimension of the searched area, $f_{\max }$ is the largest functional value that is located in the searched population, $f_{\min }$ is the smallest functional value, $\epsilon$ is the tolerance of the distance of the largest and smallest functional value, MaxIter*d is the limitation of the maximum number of permitted function evaluations during the execution of the algorithm. For test functions, where the solution to the problem is known in advance, it is sufficient that the best point of the population has a value less than f_near, a value close enough to the global minimum that we pre-set. At the end of the algorithm, we find the type of search termination.

Differential Evolution is a stochastic algorithm for a heuristic search for a global minimum using evolutionary operators [10], [1]. The Differential Evolution algorithm creates a new population $Q$ by gradually creating a point $y$ for each point $x_{i}, i=1, \ldots, N$ of the old population $P$, and assigning a point with a lower functional value to the population $Q$ from that pair. The point $y$ is created by crossing the vector $v$, where the point $v$ is generated from three different points, $r_{1}, r_{2}, r_{3}$ which are randomly selected from the population $P$ and different from the point $x_{i}$ of the relationship $v=r_{1}+F\left(r_{2}-r_{3}\right)$, where $F>0$ is the input parameter which can be determined according to different rules and the vector $x_{i}$ so that any of its elements $x_{i j}, i=1, \ldots, N, j=1, \ldots, d$, is replaced by a value $v_{j}$ with probability $C \in\langle 0,1\rangle$. If no change occurs for $x_{i j}$ or for $C=0$ one randomly selected vector $x_{i}$ element is replaced. We can see that, compared to the algorithm 
Controlled Random Search, Differential Evolution does not replace the worst point in a population but only the worse of a pair of points and thus the Differential Evolution algorithm tends to end searches in a local minimum. On the other hand, however, it converges more slowly with the same end condition. The algorithm for generating a point $y$ can be entered as a function in Matlab [12]:

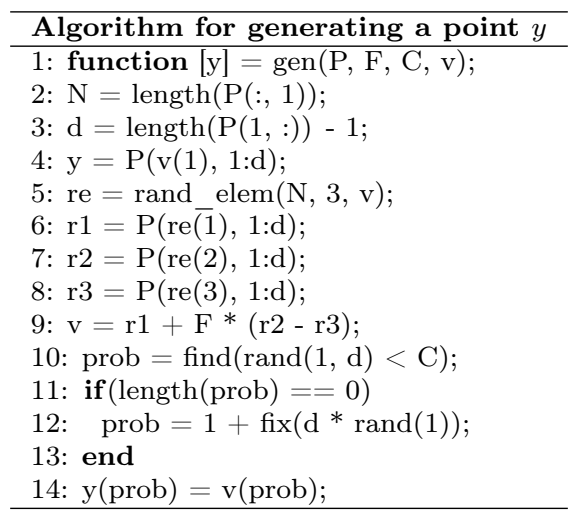

Selecting points $r_{1}, r_{2}, r_{3}$ from the population $P$ provides a function

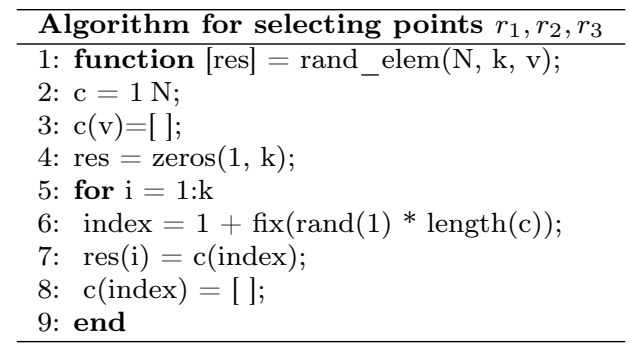

We construct the source text of the algorithm Differential Evolution in the same way as with the algorithm Controlled Random Search as the m-file difevol.m.

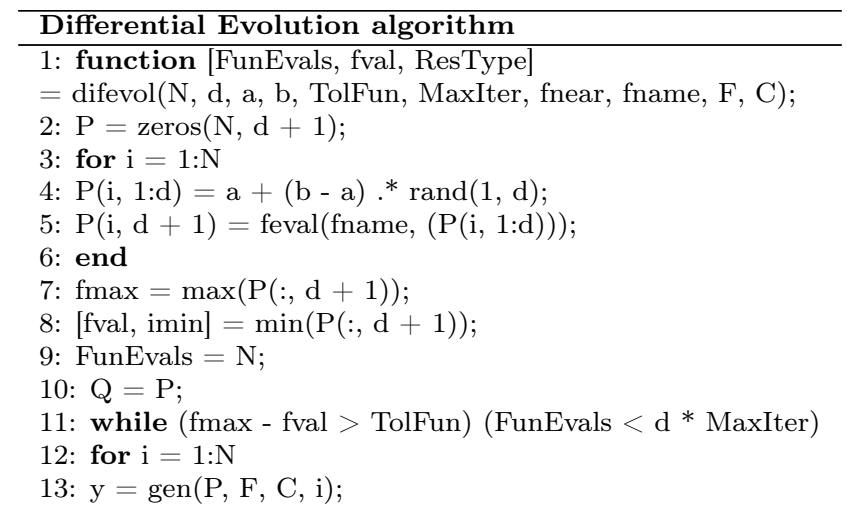




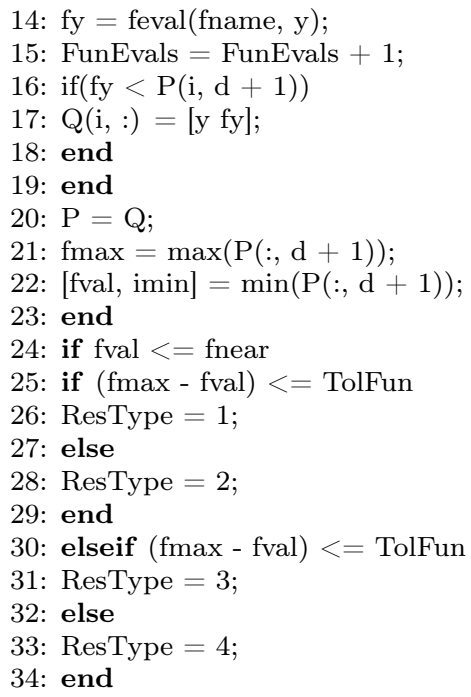

\section{Methodology of research and algorithms verifica- tion and comparison}

The experiment was carried out at the Faculty of Natural Sciences of Constantine the Philosopher University in Nitra during the academic years 2018/2019 and 2019/2020. A total of 42 students of single branch study of mathematics and students of teaching combined with maths, who selected the subject numerical mathematics, participated in the experiment. The group of students was taught a selected part "global optimization" of the mathematics curriculum with use of Matlab.

The aim of our research was to verify the behavior and efficiency of three selected algorithms in the global optimization problem. We used four known test functions to test the efficiency of each of the three selected algorithms. In the experiment while searching a global optimum, we recorded the number of evaluations of each algorithm used for each of the selected function. When algorithms are compared, tests must be performed under the same conditions. The experiment was realized for two different dimensions $d=3$ and $d=4$, the number of times the minimum search is repeated to 100 , tolerance $\epsilon$ to the value seteps $=1 \mathrm{e}-7$; and the default value MaxIter=10000 for one dimension. The search algorithm ends when the minimum and maximum distance are at the selected value or the maximum number of function evaluations has been reached. Especially for the function fminsearch, the above end condition is maintained by the code sequence:

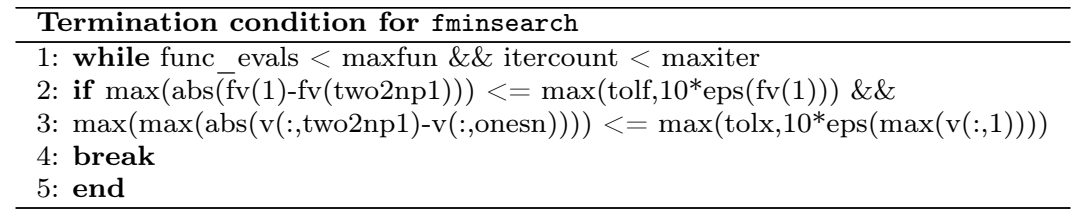


in the part Main algorithm of the source text fminsearch [6]. The func_evals variable is in the role of the variable FunEvals, the maxfun constant in the role of the expression MaxIter* $\mathrm{d}$.

The required limit to the number of algorithm iterations and the tolerance of any point in the population from the local minimum point, but also the necessary tolerance $\epsilon$ of functional values and the limit to the maximum number of test function evaluations can be adjusted by setting the appropriate parameters of the structure options for the fminsearch function.

optimset('MaxFunEvals', MaxIter*d, 'MaxIter', MaxIter*d, 'TolX', seteps, 'TolFun', seteps));

The dimension of space to be searched, the space limitations, the number of searches repeated and ensuring that the correct function is linked to the algorithm and the correct file name for storing the necessary records are entered through the formal run_test parameters that can be run for each global optimization algorithm (depending on the altype parameter). While searching for the global optimum, the function writes the repeat number, the number of function evaluations, the type of algorithm termination, the function value of the minimum found into the filenamesaveres text file.

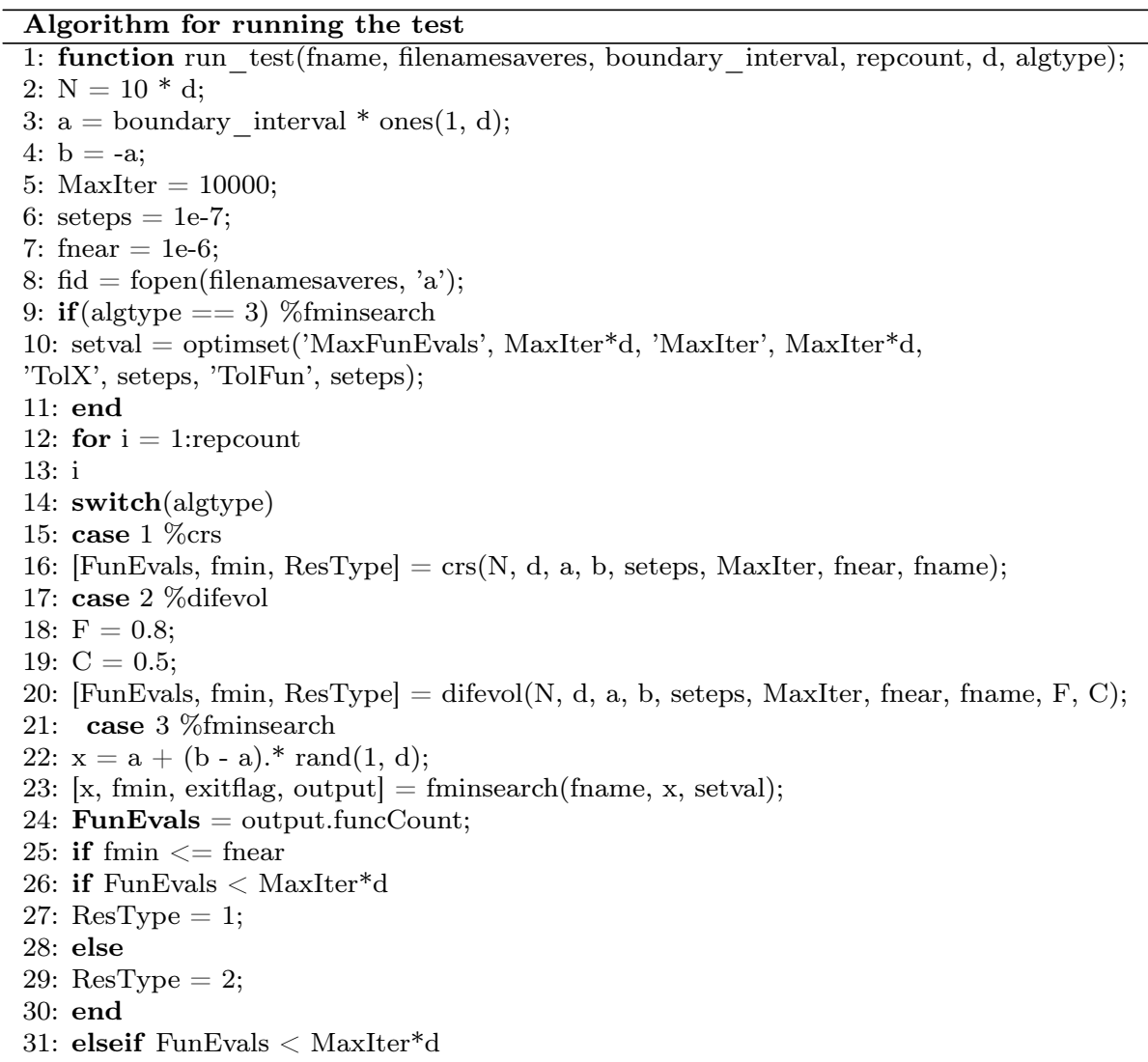


32: ResType $=3$;

33: else

34: ResType $=4$;

35: end

36: end

37: fprintf(fid, '\%5.0f ', i);

38: fprintf(fid, '\%10.0f', FunEvals);

39: fprintf(fid, '\%1.0f', ResType);

40: fprintf(fid, ' \%15.4e', fmin);

41: fprintf(fid, '\% $1 \mathrm{~s} \backslash \mathrm{r} \backslash \mathrm{n}$ ', ' ');

42: end

43: fclose(fid);

The fminsearch algorithm has 4 output parameters ( $x$, fmin, exitflag, output). Thus, in determining the type of algorithm termination for the fminsearch function, it is not possible to use the non-existent variable fmax. The disadvantage in the run_test function above is solved by using the nested funcCount element of the output structure, which indicates the number of function evaluations.

We ran the run_test function for each dimension, for each algorithm, and for each test function, so we each time got 100 evaluations of the test function by selected algorithm in selected dimension.

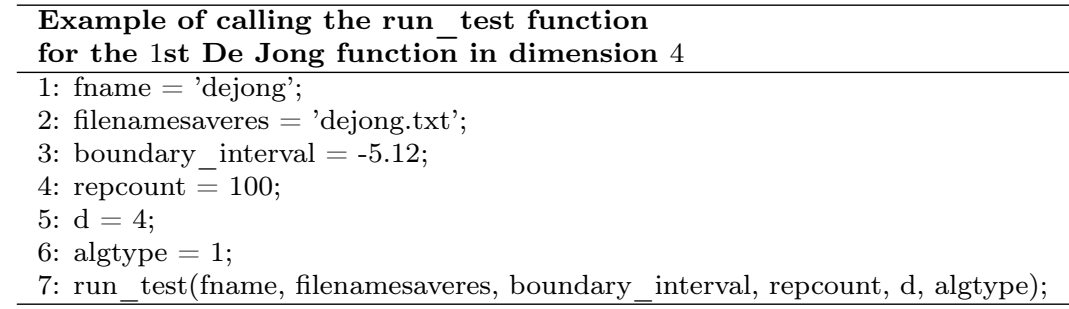

For example, program code creates a dejong.txt file with 100 rows and 4 columns i, FunEvals, ResType, fmin for the 1st De Jong function in dimension 4, with the CRS algorithm used. Thus, for all combinations of the algorithm and the test function, we get 12 files for each dimension, each with 100 evaluations.

\section{Results of the experiment and their statistical analysis}

Based on the results of the experiment, we can compare the search time complexity [2] and reliability of the algorithms used. The time complexity of the algorithm is determined by the number of test function evaluations during the search, which ensures comparability of results regardless of the speed of the computer used. We analysed the results of the experiment using selected statistical methods. Since we have been following the influence of two factors - the algorithm and function on the assessment numbers, the possibility to use a two-factor variance analysis in 
addition to descriptive statistics was offered for the assessment of the results of the experiment. However, we can only use the variance analysis if the following conditions are met: The sample files come from the basic files with normal distributions, the sample files are independent of each other and the variances of the basic files are equal. Given that the observed feature assumptions described above were not met, we used the non-parametric method of Kruskal-Wallis test [5] for the analysis. Since the Kruskal-Wallis test is a non-parametric analogue to a one-factor analysis, all combinations of the levels of the original two factors were a factor: algorithms and types of functions. In our case, we tested 3 algorithms in combination with 4 types of functions, so we gained 12 independent selections (sub-groups) or 12 levels of the factor "algorithm type + function type" in each of the two dimensions.

In the experiment, 100 measurements of the assessment numbers were performed in dimension 3 and 4 in each of the 12 selections (so-called sub-groups), i.e. altogether 1200 measurements. The tested problem is formulated as follows. We test a null hypothesis $H_{0}$ : the numbers of evaluations in the 12 sub-groups created according to the factor levels "algorithm type + function type" are identical as in the alternative hypothesis $H_{1}$ : The numbers of evaluations in the 12 sub-groups created according to the factor levels indicated are not identical (or, at least at a level, they are different). As we have already stated, since the condition of the normal distribution of observed features was not met, we used the Kruskal-Wallis test to test the null hypothesis.

The Kruskal-Wallis test is a non-parametric analogue to one-factor variance analysis, i.e. it allows testing the hypothesis $H_{0}$ that $k(k \geq 3)$ independent files originate from the same distribution. It is a direct generalization of Wilcoxon signed-rank test in the case $\mathrm{k}$ of independent selection files $(k \geq 3)$.

Let's mark $n_{1}, n_{2}, \ldots, n_{k}$ the ranges of individual selection files. Let's pose, $n=n_{1}+n_{2}+\cdots+n_{k}$. Let's line all $n$ elements into a non-decreasing sequence and let's assign its rank to each element. Let's mark $T_{i}$ the sum of the elements ranks of the ith selection file $(i=1,2, \ldots, k)$. Since $T_{1}+T_{2}+\cdots+T_{k}=\frac{n(n+1)}{2}$ must hold, we can use this relationship to check the calculation of the values of the characteristics $T_{i}(i=1,2, \ldots, k)$. The test statistics is the statistics

$$
K=\frac{12}{n(n+1)} \sum_{i=1}^{k} \frac{T_{i}^{2}}{n_{i}}-3(n+1)
$$

which has asymptotically the $\chi^{2}$-distribution with $k-1$ degrees of freedom. We reject the null hypothesis $H_{0}$ at significance level $\alpha$ if $K \geq \chi^{2}(k-1)$, where $\chi^{2}(k-1)$ is the critical value of the $\chi^{2}$-distribution with $k-1$ degrees of freedom. As the statistics $K$ has an asymptotic $\chi^{2}$-distribution, we can only use the above relationship if the selections have a large range $\left(n_{i} \geq 5, i=1,2, \ldots, k\right)$, and if $k \geq 4$. For some $i$ is $n_{i}<5$, or if $k=3$, we compare the test criteria value $K$ with the critical value $K_{\alpha}$ of the Kruskal-Wallis test. Critical values $K_{\alpha}$ are listed in the critical values table. The tested hypothesis $H_{0}$ is rejected at significance level $\alpha$ if $K \geq K_{\alpha}$. 
If identical values occur in the obtained sequence data, that are assigned the average rank, it is necessary to divide the value of the testing criterion $K$ by the so-called correction factor. Its value is calculated by the following formula:

$$
f=1-\frac{\sum_{i=1}^{p}\left(t_{i}^{3}-t_{i}\right)}{n^{3}-n}
$$

where $p$ is the number of classes with the same rank, $t_{i}$ the number of ranks in the $i$-th class. The testing statistics will then have the form

$$
K_{2}=\frac{K}{f} .
$$

If we reject the tested hypothesis $H_{0}$ in favour of the alternative hypothesis $H_{1}$, which means that the selections do not come from the same distribution, a question remains unanswered: which selections differ statistically significantly from each other. In the analysis of variance, Duncan test, Tukey method, Scheffe method or Neményi test are used to answer this question. In the Kruskal-Wallis test, Tukey method is most frequently used to test contrasts, which we also briefly describe below.

In the Tukey method, we compare the $i$-th and the $j$-th file for each $i, j$, where $i, j=1,2, \ldots, k$ and $i \neq j$, according to the following procedure. For each pair of compared files, we calculate average ranks

$$
\bar{T}_{i}=\frac{T_{i}}{n_{i}}, \quad \bar{T}_{j}=\frac{T_{j}}{n_{j}} .
$$

The testing criterion of the null hypothesis $H_{0}$, that the distributions of the files $i$ and $j$ are identical, is the absolute value of the difference in their average rank

$$
D=\left|\bar{T}_{i}-\bar{T}_{j}\right|
$$

The tested hypothesis $H_{0}$ is rejected at significance level $\alpha$, if $D>C$, where

$$
C=\sqrt{\chi_{\alpha}^{2}(k-1) \frac{n(n+1)}{12}\left(\frac{1}{n_{i}}+\frac{1}{n_{j}}\right)}
$$

$\chi_{\alpha}^{2}(k-1)$ is the critical value of the $\chi^{2}$-distribution with $k-1$ degrees of freedom, $k$ is the number of compared files. In our case, we verified by the Kruskal-Wallis test whether the 12 sub-groups produced by the level of the factor "type of algorithm + type of function" statistically significantly differ in the observed feature "the numbers of evaluations". Therefore $k=12$, while $n_{1}=n_{2}=\cdots=n_{12}=100, n=$ $n_{1}+n_{1}+\cdots+n_{1} 2=1200$ are measured numbers of evaluations. We implemented the Kruskal-Wallis test in program STATISTICA. After entering the input data in the computer output reports, we get the following results for the selected KruskalWallis test: the testing criterion value $H$ and the probability value $p$. 


\section{Dimension 3}

We have used the Kruskal-Wallis test to test the null hypothesis $H_{0}$ : the numbers of evaluations in the 12 sub-groups created according to the factor levels "algorithm type + function type" are identical as in the alternative hypothesis $H_{1}$ : the numbers of evaluations in the 12 sub-groups created according to the factor levels indicated are not identical (or, at least at a level, they are different).

First, we calculated arithmetic averages and standard deviations of the assessment numbers (Table 1) and also presented it graphically in Figure 1 in each of the 12 sub-groups.

\begin{tabular}{clcl}
\hline \multicolumn{4}{l}{ Evaluations count } \\
\hline Groups & Means & $\mathrm{N}$ & Std. Dev. \\
\hline 1 & 22709,21 & 100 & 7098,061 \\
2 & 2249,60 & 100 & 114,419 \\
3 & 27863,82 & 100 & 3143,617 \\
4 & 2703,36 & 100 & 191,001 \\
5 & 5980,20 & 100 & 328,916 \\
6 & 2711,70 & 100 & 117,233 \\
7 & 10827,00 & 100 & 1327,715 \\
8 & 16384,20 & 100 & 1092,761 \\
9 & 192,89 & 100 & 25,877 \\
10 & 234,79 & 100 & 14,931 \\
11 & 272,89 & 100 & 28,379 \\
12 & 376,47 & 100 & 69,821 \\
\hline All Grps. & 7708,84 & 1200 & 9504,885 \\
\hline
\end{tabular}

Table 1: Numbers of evaluations in each subgroup in dimension 3

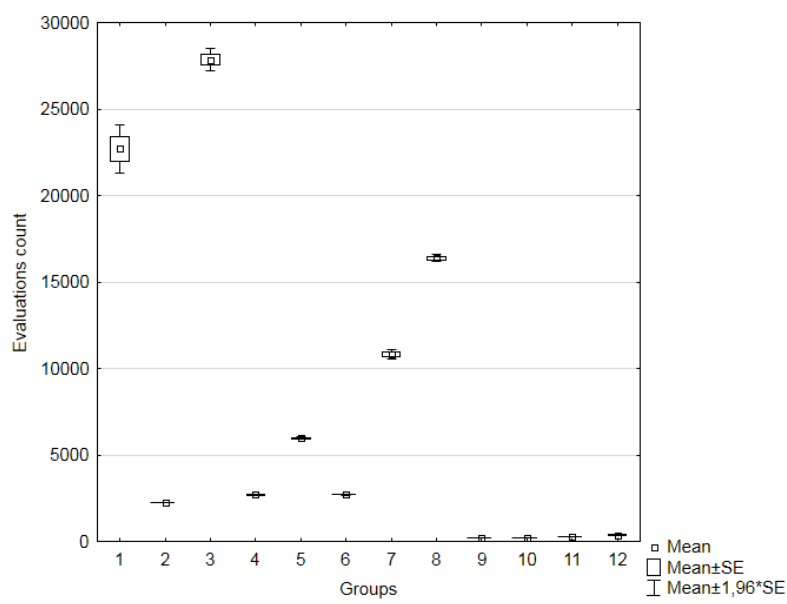

Figure 1: Numbers of evaluations (average values) in each subgroup in dimension 3

We have calculated the rank sums Table 2 , the test criterion value $K=1172.02$ 
and the value $p=0.000$ by the Kruskal-Wallis test in dimension 3 for the assessment numbers. As the calculated probability value $p$ is less than 0.01 , we reject the null hypothesis at the significance level $\alpha=0.01$, i.e. the difference between the 12 sub-groups in dimension 3 with respect to the observed feature of the "number of evaluations" is statistically significant.

\begin{tabular}{ccl}
\hline Groups & $\begin{array}{c}\text { Evaluation } \\
\text { count }\end{array}$ & $\begin{array}{l}\text { Sum of } \\
\text { Ranks }\end{array}$ \\
\hline 1 & 100 & 105274,0 \\
2 & 100 & 45221,0 \\
3 & 100 & 111855,0 \\
4 & 100 & 59602,0 \\
5 & 100 & 75050,0 \\
6 & 100 & 60327,0 \\
7 & 100 & 85271,5 \\
8 & 100 & 97799,5 \\
9 & 100 & 6294,5 \\
10 & 100 & 15348,0 \\
11 & 100 & 24279,5 \\
12 & 100 & 34278,0 \\
\hline
\end{tabular}

Table 2: Kruskal-Wallis test results

The test confirmed that the individual sub-groups in dimension 3 differ statistically significantly from each other in relation to the assessment numbers. In the same way, as in dimension 2 , we have been able to find out by multiple comparisons which groups are statistically significantly different from each other Table 3 in this case.

\begin{tabular}{|c|c|c|c|c|c|c|c|c|c|c|c|}
\hline \multicolumn{12}{|c|}{ Groups } \\
\hline & 2 & 3 & 4 & 5 & 6 & 7 & 8 & 9 & 10 & 11 & 12 \\
\hline 1 & $0,00^{*}$ & 1,00 & $0,00^{*}$ & $0,00^{*}$ & $0,00^{*}$ & $0,00^{*}$ & 1,00 & $0,00^{*}$ & $0,00^{*}$ & $0,00^{*}$ & $0,00 *$ \\
\hline 2 & & $0,00^{*}$ & 0,22 & $0,00^{*}$ & 0,14 & $0,00^{*}$ & $0,00^{*}$ & $0,00^{*}$ & $0,00^{*}$ & $0,00^{*}$ & 1,00 \\
\hline 3 & & & $0,00^{*}$ & $0,00^{*}$ & $0,00^{*}$ & $0,00^{*}$ & 0,27 & $0,00^{*}$ & $0,00^{*}$ & $0,00^{*}$ & $0,00^{*}$ \\
\hline 4 & & & & 0,11 & 1,00 & $0,00^{*}$ & $0,00^{*}$ & $0,00^{*}$ & $0,00^{*}$ & $0,00^{*}$ & $0,00^{*}$ \\
\hline 5 & & & & & 0,18 & 1,00 & $0,00^{*}$ & $0,00^{*}$ & $0,00^{*}$ & $0,00^{*}$ & $0,00^{*}$ \\
\hline 6 & & & & & & $0,00^{*}$ & $0,00^{*}$ & $0,00^{*}$ & $0,00^{*}$ & $0,00^{*}$ & $0,00^{*}$ \\
\hline 7 & & & & & & & 0,70 & $0,00^{*}$ & $0,00^{*}$ & $0,00^{*}$ & $0,00^{*}$ \\
\hline 8 & & & & & & & & $0,00^{*}$ & $0,00^{*}$ & $0,00^{*}$ & $0,00^{*}$ \\
\hline 9 & & & & & & & & & 1,00 & $0,02^{*}$ & $0,00^{*}$ \\
\hline 10 & & & & & & & & & & 1,00 & $0,01^{*}$ \\
\hline 11 & & & & & & & & & & & 1,00 \\
\hline
\end{tabular}

Table 3: Results of Kruskal-Wallis multiple comparison test ( $p$-values)

The Table 3 shows that there is a statistically significant difference in the numbers of evaluations in dimension 3 between sub-group 1 and sub-group 2, between sub-group 1 and sub-groups 4 to 7 and between the sub-group 1 and sub-groups 9 to 12 (probability value $p=0.000$ ). This means that the measured assessment 
numbers in sub-group 1 are statistically significantly different as measured in subgroup 2 and sub-groups 4 to 7 and 9 to 12 (or the assessment numbers between sub-groups 1st and 2nd and between the 1st sub-group and sub-groups 4-7 a 912 are significantly different respectively). In the same way, we can interpret all results in Table 3 marked with a * We also illustrated the situation graphically (Figure 2).

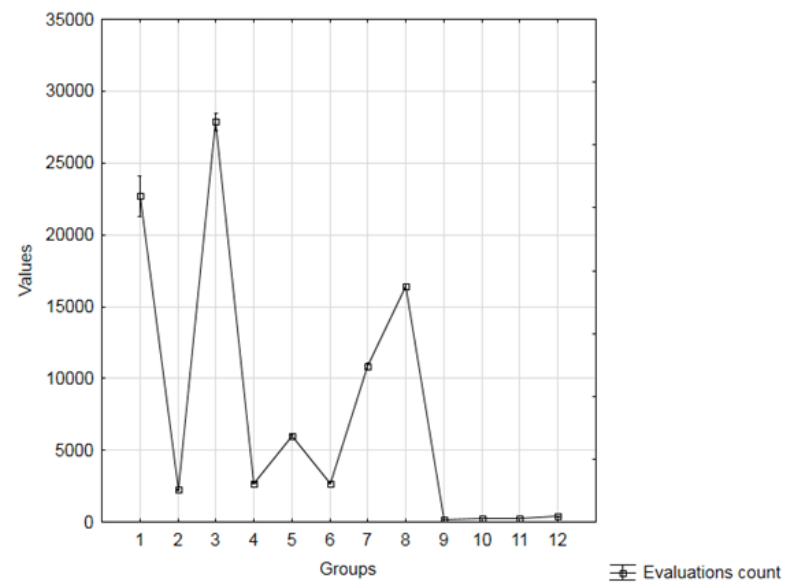

Figure 2: Numbers of evaluations (average values) in each subgroup in dimension 3

\section{Dimension 4}

As in previous dimensions, we also tested the statistical significance of differences in the number of evaluations in 12 sub-groups by the Kruskal-Wallis test in dimension 4. In each of the 12 sub-groups of dimension 4, we calculated arithmetic averages and standard deviations of the number of evaluations (Table 4) and we also presented the situation graphically in Figure 3.

We have calculated the rank sums (Table 5) and the test criterion value $K=$ 1180.46 and the value $p=0.000$ by the Kruskal-Wallis test. As the calculated probability value $p$ is as well less than 0.01 in this case, we reject the null hypothesis at the significance level $\alpha=0.01$, i.e. the difference between the 12 sub-groups in dimension 4 with respect to the observed feature "numbers of evaluations" is statistically significant. The Kruskal-Wallis test confirmed that the individual sub-groups in dimension 4 differ statistically significantly from each other in relation to the assessment numbers. Subsequently, we have identified by multiple comparisons (Table 6) which groups are statistically significantly different from each other. Table 6 shows that there is a statistically significant difference in the numbers of evaluations in dimension 4 between sub-group 1and sub-group 2. and sub-group 1 and sub-groups 4 to 6 and between the sub-group 1 and sub-groups 


\begin{tabular}{clcl}
\hline \multicolumn{4}{l}{ Evaluations count } \\
\hline Groups & Means & $\mathrm{N}$ & Std. Dev. \\
\hline 1 & 34741,03 & 100 & 3119,75 \\
2 & 4666,28 & 100 & 186,43 \\
3 & 40000,00 & 100 & 0,00 \\
4 & 5618,71 & 100 & 237,65 \\
5 & 11676,80 & 100 & 518,89 \\
6 & 4992,00 & 100 & 160,60 \\
7 & 22800,00 & 100 & 2404,70 \\
8 & 40000,00 & 100 & 0,00 \\
9 & 298,57 & 100 & 57,56 \\
10 & 379,91 & 100 & 44,35 \\
11 & 439,19 & 100 & 61,26 \\
12 & 609,18 & 100 & 129,52 \\
\hline All Grps & 13851,81 & 1200 & 15432,29 \\
\hline
\end{tabular}

Table 4: Numbers of evaluations in each subgroup in dimension 4

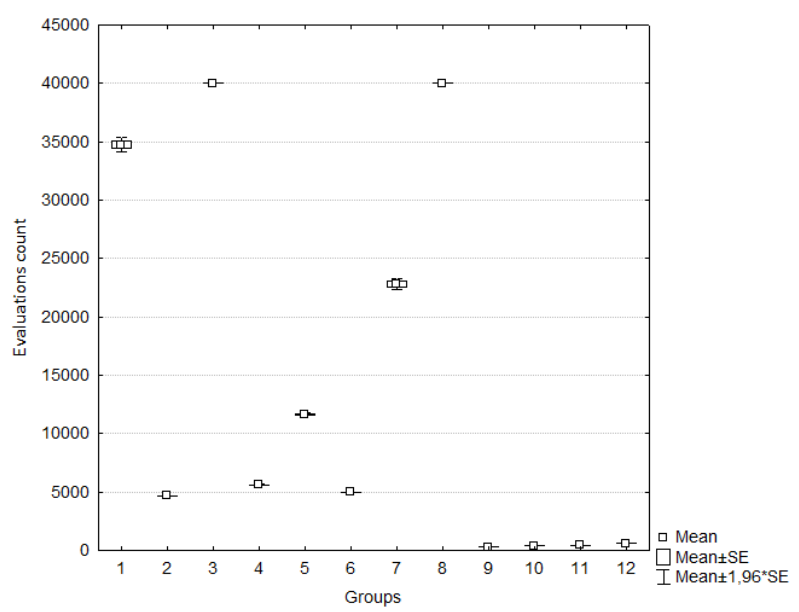

Figure 3: Numbers of evaluations (average values) in each subgroup in dimension 4

9 to 12 (probability value $p=0.000$ ). This means that the measured assessment numbers in sub-group 1 in dimension 4 are statistically significantly different as measured in this dimension in sub-groups 2, 4 to 6 as well as 9 to 12 (or the assessment numbers between sub-groups 1 and sub-groups $4-7$ and $9-12$ in dimension 4 are significantly different). In the same way, we can interpret all results in Table 6 marked with $\mathrm{a}^{*}$. We also illustrated the situation graphically (Figure 4). 


\begin{tabular}{ccl}
\hline Groups & $\begin{array}{c}\text { Evaluation } \\
\text { count }\end{array}$ & $\begin{array}{l}\text { Sum of } \\
\text { Ranks }\end{array}$ \\
\hline 1 & 100 & 105000,0 \\
2 & 100 & 49972,5 \\
3 & 100 & 105000,0 \\
4 & 100 & 65050,0 \\
5 & 100 & 75050,0 \\
6 & 100 & 50127,5 \\
7 & 100 & 85200,0 \\
8 & 100 & 105000,0 \\
9 & 100 & 5884,5 \\
10 & 100 & 16658,5 \\
11 & 100 & 23892,5 \\
12 & 100 & 33764,5 \\
\hline
\end{tabular}

Table 5: Kruskal-Wallis test results

\begin{tabular}{|c|c|c|c|c|c|c|c|c|c|c|c|}
\hline \multicolumn{12}{|c|}{ Groups } \\
\hline & 2 & 3 & 4 & 5 & 6 & 7 & 8 & 9 & 10 & 11 & 12 \\
\hline 1 & $0,00^{*}$ & 0,24 & $0,00^{*}$ & $0,00^{*}$ & $0,00^{*}$ & 1,00 & 0,24 & $0,00^{*}$ & $0,00^{*}$ & $0,00^{*}$ & $0,00^{*}$ \\
\hline 2 & & $0,00^{*}$ & $0,01^{*}$ & $0,00^{*}$ & 1,00 & $0,00^{*}$ & $0,00^{*}$ & $0,00^{*}$ & $0,00^{*}$ & $0,00^{*}$ & 0,83 \\
\hline 3 & & & $0,00^{*}$ & $0,00^{*}$ & $0,00^{*}$ & $0,00^{*}$ & 1,00 & $0,00^{*}$ & $0,00 *$ & $0,00^{*}$ & $0,00 *$ \\
\hline 4 & & & & 1,00 & 1,00 & $0,00^{*}$ & $0,00^{*}$ & $0,00^{*}$ & $0,00^{*}$ & $0,00^{*}$ & $0,00^{*}$ \\
\hline 5 & & & & & $0,00^{*}$ & 1,00 & $0,00 *$ & $0,00^{*}$ & $0,00^{*}$ & $0,00^{*}$ & $0,00^{*}$ \\
\hline 6 & & & & & & $0,00^{*}$ & $0,00^{*}$ & $0,00^{*}$ & $0,00^{*}$ & $0,00^{*}$ & $0,00^{*}$ \\
\hline 7 & & & & & & & $0,00^{*}$ & $0,00^{*}$ & $0,00^{*}$ & $0,00^{*}$ & $0,00^{*}$ \\
\hline 8 & & & & & & & & $0,00^{*}$ & $0,00^{*}$ & $0,00^{*}$ & $0,00^{*}$ \\
\hline 9 & & & & & & & & & 1,00 & $0,04^{*}$ & $0,00^{*}$ \\
\hline 10 & & & & & & & & & & 1,00 & $0,02^{*}$ \\
\hline 11 & & & & & & & & & & & 1,00 \\
\hline
\end{tabular}

Table 6: Results of Kruskal-Wallis multiple comparison test ( $p$-values)

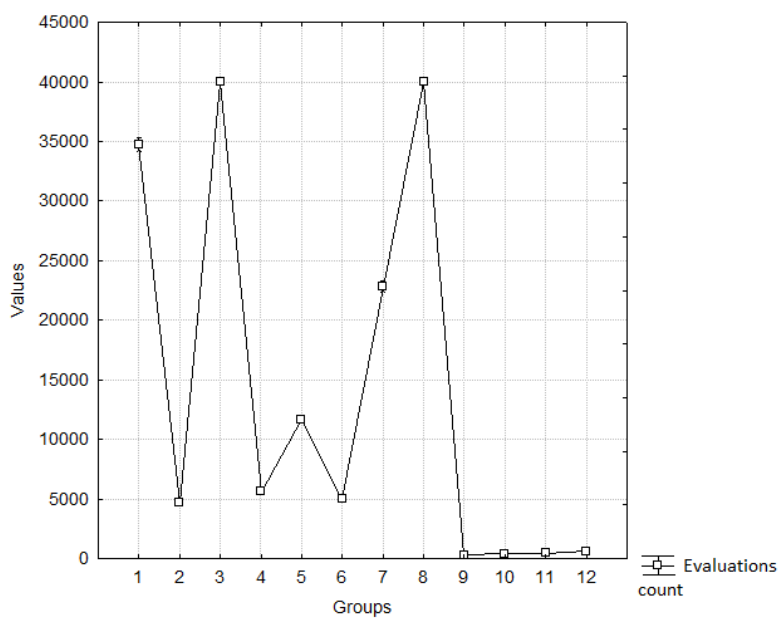

Figure 4: Numbers of evaluations (average values) in each subgroup in dimension 4 


\section{Conclusion}

In conclusion, we can summarize based on the results of the experiment that the fminsearch function is the fastest algorithm and, on the other hand, the Differential Evolution algorithm is the slowest one. Only type 1 search is considered successful. Then the reliability of finding the global minimum can be characterized as the relative number of type 1 termination, that is $R=\frac{n_{1}}{n}$, where $n_{1}$ is the number of type 1 terminations and $n$ the number of repetitions. The algorithms based on the experiment determine this reliability (in percent) of the global minimum finding (Table 7).

\begin{tabular}{lcccc}
\hline De Jong 1 & Rosen & Ackley & Griewangk & Average \\
\hline CRS & & & & \\
$\quad 100 \%$ & $100 \%$ & $53 \%$ & $3 \%$ & $64 \%$ \\
$\begin{array}{c}\text { fminsearch } \\
100 \%\end{array}$ & $87 \%$ & $0 \%$ & $0 \%$ & $47 \%$ \\
$\begin{array}{c}\text { difevol } \\
100 \%\end{array}$ & $35 \%$ & $99 \%$ & $94 \%$ & $82 \%$ \\
\hline
\end{tabular}

Table 7: Reliability of algorithms id dimensions 3 and 4

We can see a considerable difference in the reliability of the Differential Evolution algorithm (which is very slow) and algorithms Controlled Random Search and fminsearch. We can say that evolutionary operations mutation, cross-breeding, and selection are a major benefit of reliability for the algorithm. The difference in reliability of the algorithm Controlled Random Search and Fminsearch can also be considered significant given the number of times the global minimum search and the test functions used are repeated. Furthermore, based on the results of the test, we can say that finding a global minimum of the First De Jong function is simple and almost certain for any algorithm. Finding a global minimum for Rosenbrock's saddle is not easy just for an algorithm Differential Evolution that searches unreliably. From the above-mentioned reliabilities, it can be said that finding the global minimum of the Ackley function and Griewangk's function is difficult for the truly fast Fminsearch algorithm implemented in the Matlab environment, which produces great results for the Second De Jong function reliably and quickly. In general, the finding of the global minimum of the Griewangk's function is least likely in dimensions 3 and 4 .

Based on the results of the experiment, we can conclude that by involving mathematical software to solve global optimization problems, a higher level of knowledge was achieved, a better understanding of various principles and algorithms, and, thus, students better mastered the issue. It is therefore effective and necessary to pay sufficient attention to these methods. Thanks to the use of computer techniques in the pedagogical process, everyone can draw into mathematics secrets of global optimization problems. On the basis of the results and theoretical starting points of the work, we have arrived at the following recommendations:

1. lead the students in solving mathematical application tasks in order to best 
understand the theoretical starting points of the subject topic

2. use computer technology to increase students' activity and to provide a successful motivation to work

3. create suitable, modern and pregnant study material that will enhance the knowledge of students

4. within the cross-subject relations, extend the students' knowledge from the computer algebra systems

5. involve the use of computer algebra systems into maths teaching for achieving better results

6. effectively use the subject matter from another field within the framework of cross-subject relationships (such as mathematics and informatics).

\section{References}

[1] Y. Gao, K. Wang, C. Gao, Y. Shen, T. Li: Application of Differential Evolution Algorithm Based on Mixed Penalty Function Screening Criterion in Imbalanced Data Integration Classification, Mathematics 7.12 (2019), p. 1237, DOI: https://doi.org/10.3390/math7121237.

[2] M. R. Garey, D. S. Johnson: Computers and intractability, vol. 174, Freeman San Francisco, 1979.

[3] K. N. Kaipa, D. Ghose: Glowworm swarm optimization: theory, algorithms, and applications, vol. 698, Springer, 2017, DOI: https://doi.org/10.1007/978-3-319-51595-3.

[4] V. Kvasnicka, J. Pospíchal, P. Tino: Evolutionary algorithms, STU Bratislava (2000).

[5] D. Markechová, B. Stehlíková, A. Tirpáková: Statistical Methods and their Applications. FPV UKF in Nitra, 534 p, 2011.

[6] Mathworks: Online documentation, accessed 6th March, 2020, 2020, URL: https://www . mathworks. com/help/matlab/ref/fminsearch.html.

[7] Mathworks: Online documentation, accessed 16th March, 2020, 2020, URL: https://www. mathworks.com/help/matlab/ref/optimset.html.

[8] S. MíkA: Mathematical optimizatio, Plzeň: ZCU Plzeň, 1997.

[9] J. A. Nelder: A Simplex Method for Function Minimization, Computer Journal 7.1 (1964), pp. 308-313.

[10] K. Price, R. M. Storn, J. A. Lampinen: Differential evolution: a practical approach to global optimization, Springer Science \& Business Media, 2006.

[11] W. L. Price: A Controlled Random Search Procedure for Global Optimization, Computer Journal 20.4 (1977), pp. 367-370.

[12] J. Tvrdík: Evolutionary algorithms, Ostrava: University of Ostrava, 2010. 